\title{
NUMERICAL ANALYSIS OF THE AB INITIO COMPUTATION OF THE EFFECTS OF IONIZATION ON THE NONLINEAR SUSCEPTIBILITY COEFFICIENTS OF THE HYDROGEN ATOM*
}

\author{
PATRICK O. KANO ${ }^{\dagger}$, MOYSEY BRIO $¥$, AND JEROME V. MOLONEY
}

\begin{abstract}
This paper provides a numerical analysis of a procedure for determining the effects of ionization on the nonlinear susceptibility coefficients of the hydrogen atom. To solve the relevant system of Schrödinger-type equations, we have developed a multidomain pseudospectral code with high accuracy symmetric finite differences to update cell boundary points. Using a conservative time stepping, one is then able to resolve the oscillatory solutions to the underlying equations, compute the ionization probability, and accurately determine the polarization. To gain insight into the physical mechanisms involved, we have calculated the full susceptibility and one which depends solely on the electronic bound-bound transitions. Our analysis reveals that saturation of the susceptibility occurs without including bound-continuum transitions. We have also found that a linear extrapolation of the total susceptibility versus the ionization probability to obtain the instantaneous susceptibility is quantitatively unreliable.
\end{abstract}

Key words. Numerical analysis, nonlinear susceptibility, time dependent Schroedinger equation

Mathematics Subject Classification 2000. 78A60, 81 V80

\section{Introduction}

While the numerical simulation of the Schrödinger equation for the hydrogen atom is a well-established field [2, 15, 24, 32], application to the computation of nonlinear susceptibility coefficients is a new development. In recent papers, Nurhuda, et. al. [30, 31] have proposed an algorithm for these coefficients based on an ab initio determination of the atom's response to an incident electric field. This has required the solution of an auxiliary set of differential equations to the Schrödinger equation and a linear extrapolation of the susceptibility to the limit of zero ionization probability. Furthermore, they demonstrate that both the total and instantaneous part of the susceptibility saturate at high intensities. In this paper, we investigate the robustness of this novel procedure and show that to gain quantitatively accurate values requires special consideration.

Our analysis has required the resolution of three main issues. First, due to the sensitivity of the procedure and the demands on computing resources for a full simulation of the Schrödinger equation, we have developed an accurate pseudospectral code. Our approach utilizes a multidomain Legendre basis in space, Strang splitting in time, and Gaussian quadrature to accurately determine spatial integrals [4, 5, 21, 40]. The second problem is to separate the effects of ionization on the nonlinear susceptibility from those due to high order terms in an expansion of the susceptibility with respect to the intensity. This has involved the numerical evaluation of the procedure for the instantaneous susceptibility and the introduction of a second polarization due solely to

\footnotetext{
*Received: August 11, 2005; accepted (in revised version): December 1, 2005. Communicated by David Cai.

${ }^{\dagger}$ Arizona Center for Mathematical Sciences, Department of Mathematics, University of Arizona, 617 N. Santa Rita, Tucson, AZ 85721, USA (pkano@math.arizona.edu).

$\ddagger$ Arizona Center for Mathematical Sciences, Department of Mathematics, University of Arizona, 617 N. Santa Rita, Tucson, AZ 85721, USA (brio@math.arizona.edu).

$\S$ Arizona Center for Mathematical Sciences, Department of Mathematics, University of Arizona, 617 N. Santa Rita, Tucson, AZ 85721, USA (jml@acms.arizona.edu).
} 
the bound-bound transitions of the electron. The last topic is an interpretation of the results of our simulations and, when possible, a comparison with analytic predictions.

The structure of the paper is thus organized as follows. Section two reviews the underlying physical description of nonlinear susceptibility and reiterates the procedure introduced by Nurhuda, et. al. [30]. In section three, we describe the numerical approach we have adopted to solve the Schrödinger equation. The fourth section contains the majority of our sensitivity analysis and results. Finally, in the last portion, we review our findings and draw conclusions.

\section{Theory}

In this section, we provide the theoretical framework for our analysis. First, we review the linear phenomenological relationship which exists for low intensity light between the susceptibility $\chi(\omega)$, the electric field $E(t)$, and the atomic dipole polarization $P(t)$. Second, we reiterate the approach by Nurhuda [31] to determine the nonlinear susceptibility coefficients which become important at high intensities.

2.1. Low intensity phenomenological description. In this paper, we utilize a semi-classical approach to the atom-light interaction. Throughout, the relevant quantities are expressed in terms of atomic units $(\hbar=c=e=1)$ [28]. In particular, we assume a classical electric field $E(t)$ but make use of quantum states within the atom.

At low light intensities $\left(I<<5 \cdot 10^{13} \mathrm{~W} / \mathrm{cm}^{2}\right)$, the description of the interaction of light with the hydrogen atom is well characterized as linear and shift-invariant. The response of the atom, its polarization $\vec{P}(t)$, to a weak external electric field is thus described by a temporal convolution $[26,27]$

$$
\vec{P}(t)=\int_{0}^{t} \bar{\chi}(t-\tau) \vec{E}(\tau) d \tau \text {. }
$$

The three dimensional vectors $\vec{P}$ and $\vec{E}$ necessitate that the time-dependent Green's function $\bar{\chi}(t)$ is a 4 th rank tensor. For cases with spherical symmetry, such as the hydrogen atom, one may replace the tensor by a scalar quantity $\chi(t)$. Furthermore, if $\vec{E}$ is linearly polarized so that $\vec{E}(t)=E(t) \hat{z}$, then the vector equation can be replaced with a scalar variant

$$
P(t)=\int_{0}^{t} \chi(t-\tau) E(\tau) d \tau
$$

The scalar temporal convolution can be transformed into a product by means of a Laplace transform

$$
\hat{P}(s)=\chi(s) \hat{E}(s)
$$

or more commonly for $\omega \in \mathbb{R}$

$$
\chi(\omega)=\lim _{\epsilon \rightarrow 0} \frac{\hat{P}(\epsilon-i \omega)}{\hat{E}(\epsilon-i \omega)} .
$$

The previous formalism is strictly correct only for a linear and shift invariant system. Often however we wish to extend it to problems where the response to a polarized electric field is nonlinear 


$$
\begin{aligned}
P(t)= & \int_{0}^{t} \chi^{(1)}(\tau) E(t-\tau) d \tau \\
& +\int_{0}^{t} \int_{0}^{t} \int_{0}^{t} \chi^{(3)}\left(t, \tau_{1}, \tau_{2}, \tau_{3}\right) E\left(\tau_{1}\right) E\left(\tau_{2}\right) E\left(\tau_{3}\right) d \tau_{1} d \tau_{2} d \tau_{3}+\cdots .
\end{aligned}
$$

For systems with inversion symmetry, such as the hydrogen atom, the even indexed susceptibility coefficients are zero.

The multiple integrals can be reduced to a single integration over time by assuming that the nonlinear susceptibility consists of a convolution term of the form [35]

$$
\chi^{(3)}\left(t, \tau_{1}, \tau_{2}, \tau_{3}\right) \Rightarrow \chi^{(3)}\left(\tau_{2}-\tau_{3}\right) \delta\left(\tau_{1}-\tau_{3}\right) \delta\left(t-\tau_{2}\right) .
$$

Substituting this form for the nonlinear susceptibility into the definition of the polarization yields

$$
P(t)=\int_{0}^{t} \chi^{(1)}(t-\tau) E(\tau) d \tau+\int_{0}^{t} \chi^{(3)}(t-\tau) E^{2}(\tau) d \tau \cdot E(t)+\cdots
$$

At this point, in order to gain a phenomenological relationship between $\hat{P}(\omega)$ and $\hat{E}(\omega)$, one makes use of the fact that the intensity of an electric field $I(\omega)$ is proportional to the maximum of the square of the field

$$
P(t) \propto \int_{0}^{t} \chi^{(1)}(t-\tau) E(\tau) d \tau+\int_{0}^{t} \chi^{(3)}(t-\tau) \delta(\tau) d \tau \cdot I(\omega) E(t)+\cdots .
$$

Laplace transforming this last expression and assuming $s=-i \omega$ yields

$$
\hat{P}(\omega) \propto\left[\chi^{(1)}(\omega)+\chi^{(3)}(\omega) I(\omega)+\cdots\right] \hat{E}(\omega) .
$$

Effectively, $\chi(\omega)$ has been expanded into a Taylor series around the intensity $I$ for a fixed frequency $\omega$

$$
\chi(\omega)=\chi^{(1)}(\omega)+\chi^{(3)}(\omega) I(\omega)+O\left(I^{3}\right) .
$$

2.2. High intensity phenomenological description. Up to this point, we have not considered the possible effects that ionization may have on the polarization or susceptibility. For low intensity light, little ionization occurs and the observed nonlinear phenomena arise from the bound electrons in the atom. As the intensity increases however ionization of the atom can be appreciable. To navigate the computation of the susceptibility coefficients for the high intensity case, Nurhuda and coworkers introduced the following approach.

In the case that the electron has been liberated, the system is no longer linear in time and the convolution relationship between $P(t)$ and $E(t)$ is invalid. Instead one would expect a complicated time dependence

$$
P(t)=\int_{0}^{\infty} G(t, \tau) E(\tau) d \tau
$$

In the low intensity limit, the more general Green's function $G(t, \tau)$ must be the convolution relation in equation (2.5). This fact suggests that the relationship between $\hat{P}(\omega)$ and $\hat{E}(\omega)$ be replaced by

$$
\chi(\omega, t) \equiv \frac{\hat{P}(\omega, t)}{\hat{E}(\omega, t)}
$$


where

$$
\begin{aligned}
\hat{P}(\omega, t) & =\int_{0}^{t} P(\tau) e^{-i \omega \tau} d \tau \\
\hat{E}(\omega, t) & =\int_{0}^{t} E(\tau) e^{-i \omega \tau} d \tau .
\end{aligned}
$$

A revealing form for this total susceptibility $\chi(\omega, t)$ can be gained by expanding the nonlinear terms of the Taylor series in equation (2.10) in terms of the ionization probability $Q(t)$.

$$
\begin{gathered}
\chi(\omega)=\chi^{(1)}(\omega)+\chi^{(3)}(Q, \omega) \cdot I(\omega)+\chi^{(5)}(Q, \omega) \cdot I^{3}(\omega)+O\left(I^{5}\right) \\
\chi(\omega)=\chi^{(1)}+\left(\chi_{0}^{(3)}+\frac{d \chi^{(3)}}{d Q} Q\right) I+\left(\chi_{0}^{(5)}+\frac{d \chi^{(5)}}{d Q} Q\right) I^{3}+O\left(I^{5}\right)+O\left(Q^{2} I\right) \\
\chi(\omega)=\left(\chi^{(1)}+\chi_{0}^{(3)} I+\chi_{0}^{(5)} I^{3}\right)+\left(\frac{d \chi^{(3)}}{d Q} I+\frac{d \chi^{(5)}}{d Q} I^{3}\right) Q+O\left(I^{5}\right)+O\left(Q^{2} I\right)
\end{gathered}
$$

This last equation can be recast into a definition which mirrors the one for the low intensity case

$$
\chi(\omega, t)=\chi^{(1)}(\omega)+\Delta \alpha(I, \omega)+\Delta \chi(Q(t), I)
$$

where

$$
\begin{array}{r}
\Delta \alpha(I, \omega)=\chi_{0}^{(3)}(\omega) I+\chi_{0}^{(5)}(\omega) I^{3}+O\left(I^{5}\right) \\
\Delta \chi(Q, I)=\left(\frac{d \chi^{(3)}}{d Q} I+\frac{d \chi^{(5)}}{d Q} I^{3}\right) Q+O\left(Q^{2} I\right) .
\end{array}
$$

An important test of the previous assumptions for the form of the susceptibility is that $\chi^{(1)}(\omega)$ should be constant for any excitation with that carrier frequency. Furthermore, the instantaneous nonlinear susceptibility $\Delta \alpha(I)$ should be obtainable from the limit of zero ionization probability

$$
\Delta \alpha(I ; \omega)=\lim _{Q(t) \rightarrow 0}\left(\chi(\omega, t)-\chi^{(1)}(\omega)\right) .
$$

This definition for $\chi(\omega, t)$ is not unique nor necessarily the most informative. Thus, we also introduce here a bound susceptibility due only to the polarization computed from the electronic states bound to the atom

$$
\chi_{b}(\omega, t) \equiv \frac{\hat{P}_{b o u n d}(\omega, t)}{\hat{E}(\omega, t)} .
$$

Since it does not depend on the continuum states, a comparison between this quantity and the total susceptibility can reveal information about ionization induced effects. Furthermore, this quantity has the advantage that the linear susceptibility $\chi_{b}^{(1)}(\omega)$ 
can be computed exactly $[7,26]$

$$
\begin{array}{r}
\chi_{b}^{(1)}(\omega)=\sum_{n=2}^{\infty} \frac{f_{n}}{\omega_{n}^{2}-\omega^{2}} \\
\omega_{n}=\frac{1}{2}\left(1-\frac{1}{n^{2}}\right) \\
f_{n}=\frac{2 \omega_{n}}{3}\left|\int_{0}^{\infty} S_{10} r S_{n 1} d r\right|^{2}
\end{array}
$$

where the function $S_{n, l}$ is the usual shell amplitude of the hydrogen bound state $\phi_{n, l, m}$

$$
\begin{array}{r}
\phi_{n, l, m}(\vec{r})=\frac{S_{n, l} Y_{l, m}}{r} \\
S_{n, l}=r^{l+1} e^{-r / n}\left(\frac{2}{n}\right)^{l} \frac{2}{n^{2}} \sqrt{\frac{(n-l-1) !}{(n+l) !}} L_{n-l-1}^{2 l+1}(2 r / n)
\end{array}
$$

$n, l, m$ are the usual quantum numbers and $L_{n}^{a}(x)$ are the generalized Laguerre polynomials [9]. Using Mathematica [41], the sum can be easily computed to high precision.

2.3. Quantum mechanics model. The previous phenomenological discussion of $\chi(\omega)$ has presupposed that the polarization $P(t)$ is a known quantity. This can be measured in an experimental situation or, if the system is properly modeled, computed numerically. In this paper, we follow works $[14,15,24]$ and solve the Schrödinger equation to compute $P_{\text {bound }}(t)$ and $P(t)$.

In the quantum mechanical description, the dipole polarization $P(t)$ for a single hydrogen atom under the influence of a linearly polarized electric field is

$$
P(t)=\langle\Psi|\vec{r} \cdot \hat{z}| \Psi\rangle
$$

where $\Psi$ is the solution to the time-dependent Schrödinger equation

$$
i \frac{\partial \Psi}{\partial t}=H \Psi
$$

The inner product in spherical coordinates is defined as

$$
\left\langle\psi_{a} \mid \psi_{b}\right\rangle=\int_{0}^{2 \pi} \int_{0}^{\pi} \int_{0}^{\infty} \psi_{a}(\vec{r})^{*} \psi_{b}(\vec{r}) r^{2} \sin (\theta) d r d \theta d \phi .
$$

For the hydrogen atom interacting with light, the Hamiltonian operator $H$ consists of two components $H_{\text {atom }}$ and $H_{\text {interaction }}$. The atomic Hamiltonian describes the atom in the absence of the external field while the interaction Hamiltonian incorporates the coupling of the field to the atom. Assuming the atom to be spherically symmetric, the atomic Hamiltonian can be written

$$
\begin{array}{r}
H_{\text {atom }}=H_{r}+\frac{l(l+1)}{2 r^{2}}+V(r) \\
H_{r}=-\frac{1}{2 r^{2}} \frac{d}{d r}\left(r^{2} \frac{d}{d r}\right)
\end{array}
$$

where $V(r)$ is a radially dependent atomic potential. Including only the Coulomb interaction of the proton and electron, $V(r)=-\frac{1}{r}$. For the interaction Hamiltonian, we assume that it is described by the time-dependent potential

$$
H_{i}=-\vec{r} \cdot \vec{E}(t) .
$$


If the electric field is polarized $\vec{E}(t)=E(t) \hat{z}$, then $H_{i}=-r \cos (\theta) E(t)$.

The full three-dimensional simulation of the Schrödinger equation is computationally demanding and not necessary for the hydrogen atom. Due to its spherical symmetry, an effective one-dimensional simulation of the radial dynamics is sufficient. Following de Vries [14], we assume that $\Psi$ can be written as a product of a radial function $\frac{R_{l, m}}{r}(r, t)$ and the spherical harmonics $Y_{l, m}(\theta, \phi)$.

$$
\Psi=\sum_{l=0}^{\infty} \sum_{m=-l}^{l} \frac{R_{l, m}}{r} Y_{l, m}
$$

The function $\frac{R_{l, m}}{r}$ is the projection of the total wave function onto the $Y_{l, m}$ angular momentum basis state. Furthermore, in the absence of an external excitation, $R_{l, m}$ is the usual shell amplitude familiar from the analytic solution of the unperturbed hydrogen atom [9]. Assuming that the incoming light is linearly polarized, $m=0$ is the only relevant $m$ index and $R_{l, m}$ is fully characterized by a single variable $R_{l}$.

To determine an equation for $R_{l}$, one substitutes assumption (2.34) into the Schrödinger equation

$$
\begin{array}{r}
H \Psi=\sum_{l}\left(H_{r}+\frac{l(l+1)}{2 r^{2}}+V(r)\right) \frac{R_{l}}{r} Y_{l, 0}+H_{i} \sum_{l} \frac{R_{l}}{r} Y_{l, 0} \\
H \Psi=\sum_{l}\left(H_{r}+\frac{l(l+1)}{2 r^{2}}+V(r)\right) \frac{R_{l}}{r} Y_{l, 0}-r E(t) \cos (\theta) \sum_{l} \frac{R_{l}}{r} Y_{l, 0}
\end{array}
$$

and taking the inner product of the resulting equation with $Y_{i, 0}$ over the angular coordinates

$$
\begin{array}{r}
\left\langle\sum_{l} i \frac{\partial}{\partial t} \frac{R_{l}}{r} Y_{l, 0}, Y_{l, 0}\right\rangle_{\theta, \phi} \\
=\sum_{l} H_{r} \frac{R_{l}}{r}\left\langle Y_{l, 0}, Y_{l, 0}\right\rangle_{\theta, \phi}+\sum_{l}\left\langle\frac{l(l+1)}{2 r^{2}} \frac{R_{l}}{r} Y_{l, 0}, Y_{l, 0}\right\rangle_{\theta, \phi} \\
+\sum_{l}\left\langle V(r) \frac{R_{l}}{r} Y_{l, 0}, Y_{l, 0}\right\rangle_{\theta, \phi}-r E(t) \sum_{l} \frac{R_{l}}{r}\left\langle Y_{l, 0}|\cos (\theta)| Y_{l, 0}\right\rangle_{\theta, \phi} .
\end{array}
$$

The dipole approximation has been used to bring the electric field $E(t)$ outside the spatial integral. To evaluate further, one can make use of the analytic expression for the angular momentum coupling coefficients with $m=0$

$$
C_{l, i}=\left\langle Y_{l}|\cos (\theta)| Y_{i}\right\rangle= \begin{cases}\frac{l+1}{\sqrt{(2 l+3)(2 l+1)}}, & i=l+1 \\ \frac{l}{\sqrt{(2 l+1)(2 l-1)}}, & i=l-1 \\ 0, & \text { else }\end{cases}
$$

To avoid coupling to states which are not computed $C_{0,-1}=C_{N_{l}, N_{l}+1}=0$.

Finally, using the orthogonality of the spherical harmonics yields the desired radial equation

$$
i \frac{\partial R_{l}}{\partial t}=-r E(t)\left(C_{l+1} R_{l+1}+C_{l-1} R_{l-1}\right)-\frac{1}{2} \frac{\partial^{2} R_{l}}{\partial r^{2}}+\left(\frac{l(l+1)}{2 r^{2}}+V(r)\right) R_{l} .
$$


The resulting numerical problem to solve is a system of equations parameterized by $l$

$$
\frac{\partial R_{l}}{\partial t}=\frac{i}{2} \frac{\partial^{2} R_{l}}{\partial r^{2}}-i \tilde{V}_{l}(r) R_{l}+i r E(t)\left(C_{l+1} R_{l+1}+C_{l-1} R_{l-1}\right)
$$

with an effective potential $\tilde{V}_{l}(r)=\frac{l(l+1)}{2 r^{2}}+V(r)$. The actual details of the numerical solution are discussed in section (3). However, assuming that $\Psi$ is known, one can determine the ionization probability $Q(t)$, polarization $P(t)$, and susceptibility $\chi(\omega)$, as follows.

The wave function $\Psi$ lives in the complete Hilbert space with orthogonal bases consisting of discrete and continuous states. For hydrogen, the bound states are known analytically and thus one can, in principle, explicitly compute the ionization probability $Q(t)$ by the equation[19]

$$
\begin{array}{r}
Q(t)=1-\sum_{n=1}^{\infty} \sum_{l=0}^{n-1}\left|\left\langle\phi_{n, l}(\vec{r}) \mid \Psi(r, t)\right\rangle\right|^{2} \\
Q(t)=1-\sum_{n=1}^{\infty} \sum_{l=0}^{n-1}\left|a_{n l}\right|^{2} .
\end{array}
$$

However, utilizing the ansatz for $\Psi$ (2.34), a computationally more efficient expression is derivable.

$$
\begin{array}{r}
\Psi=\Psi_{b}+\Psi_{i}=\sum_{l=0}^{\infty} \frac{R_{l}}{r} Y_{l, 0} \\
\Psi_{b}=\sum_{n=1}^{\infty} \sum_{k=0}^{k<n} a_{n, k} \phi_{n, k}=\sum_{n=1}^{\infty} \sum_{k=0}^{k<n} a_{n, k} \frac{S_{n, k}}{r} Y_{k, 0} \\
\Psi_{i}=\sum_{l=0}^{\infty} \frac{R_{l}}{r} Y_{l, 0}-\sum_{n=1}^{\infty} \sum_{k=0}^{k<n} a_{n, k} \frac{S_{n, k}}{r} Y_{k, 0}
\end{array}
$$

Taking the inner product over the angular coordinates of this last equation with $Y_{q, 0}$

$$
\oint Y_{q, 0} \Psi_{i} d \Omega=\sum_{l=0}^{\infty} \frac{R_{l}}{r} \oint Y_{q, 0} Y_{l, 0} d \Omega-\sum_{n=1}^{\infty} \sum_{k=0}^{k<n} a_{n, k} \frac{S_{n, k}}{r} \oint Y_{q, 0} Y_{k, 0} d \Omega
$$

and using the orthonormality of the spherical harmonics reveals that the radial functions $R_{l}(r)$ can be expressed as a sum of two simple terms

$$
R_{q}(r)=\sum_{n=q+1}^{\infty} a_{n, q}(t) S_{n, q}(r)+r \oint Y_{q, 0} \Psi_{i}(\vec{r}) d \Omega .
$$

The expression for $a_{n, q}$ can be obtained by applying the spatial part of the inner product

$$
\begin{aligned}
& a_{n, q}=\int_{0}^{\infty} S_{n, q} R_{q} d r-\int_{0}^{\infty} \oint \frac{Y_{q, 0} \cdot S_{n, q}}{r} \Psi_{i}(\vec{r}) r^{2} d r d \Omega \\
& a_{n, q}=\int_{0}^{\infty} S_{n, q} R_{q} d r-\int_{0}^{\infty} \oint \phi_{n, q} \Psi_{i}(\vec{r}) r^{2} d r d \Omega \\
& a_{n, q}=\int_{0}^{\infty} S_{n, q} R_{q} d r .
\end{aligned}
$$


The last step is justified by the orthogonality of the ionized and bound parts of $\Psi$. To compute the ionization probability therefore requires an integration in one variable for each coefficient.

An expression for the polarization $P(t)$ can be derived by substituting the expansion for $\Psi$ into the definition $P(t)=\langle\Psi|r \cos (\theta)| \Psi\rangle$.

$$
\begin{array}{r}
P(t)=\left\langle\sum_{l} \frac{R_{l} Y_{l, 0}}{r}|r \cos (\theta)| \sum_{i} \frac{R_{l} Y_{l, 0}^{\prime}}{r}\right\rangle \\
P(t)=\sum_{l, i}\left\langle\frac{R_{l}}{r}|r| \frac{R_{l}}{r}\right\rangle\left\langle Y_{l, 0}|\cos (\theta)| Y_{l, 0}\right\rangle \\
P(t)=\sum_{l}\left(C_{l, l+1}\left\langle\frac{R_{l}}{r}|r| \frac{R_{l+1}}{r}\right\rangle+C_{l, l-1}\left\langle\frac{R_{l}}{r}|r| \frac{R_{l-1}}{r}\right\rangle\right) \\
P(t)=\sum_{l=0}^{L} \int_{0}^{\infty} R_{l}^{*}\left(C_{l, l+1} R_{l+1}+C_{l, l-1} R_{l-1}\right) r d r \\
P(t)=2 \sum_{l=0}^{L-1} \int_{0}^{\infty} C_{l, l+1} \Re\left(R_{l}^{*} R_{l+1}\right) r d r
\end{array}
$$

It is important to note that this total polarization $P(t)$ includes contributions from the bound-to-bound and the bound-to-continuum transitions.

However, if one considers equation $(2.47)$ for $R_{l}(r)$, it is clear that a new radial function which depends solely on the bound states can also be defined

$$
\rho_{l}(r)=\sum_{n=l+1}^{\infty} a_{n, l} S_{n, l} .
$$

The bound polarization $P_{b o u n d}(t)$ in the calculation of $\chi_{b}(\omega)$ from section $(2.2)$ is then given by

$$
P_{\text {bound }}(t)=2 \sum_{l=0}^{L-1} \int_{0}^{\infty} C_{l, l+1} \Re\left(\rho_{l}^{*} \rho_{l+1}\right) r d r .
$$

2.4. Practical sensitivity issues. While the approach of the previous sections is correct from an analytic perspective, it may be numerically unrealizable.

First to consider is the error to the polarization $P(t)$ from a small radially dependent perturbation $\epsilon(r):[0, \infty) \rightarrow \mathbb{C}$ to each $R_{l}$ solution

$$
\tilde{R}_{l}=R_{l}+\epsilon_{l}(r) \text {. }
$$

This type of additive noise can be easily generated by an explicit time stepping method with inherent damping. It can also be generated by finite domain effects such as unphysical reflections and truncated infinite integrals. The computed polarization is then

$$
\begin{array}{r}
\tilde{P}=2 \sum_{l=0}^{\infty} C_{l+1} \int_{0}^{\infty} \Re\left(\tilde{R}_{l}^{*} \tilde{R}_{l+1}\right) r d r \\
\tilde{P}=2 \sum_{l=0}^{\infty} C_{l+1} \int_{0}^{\infty} \Re\left(R_{l}^{*} R_{l+1}+\epsilon_{l}^{*} R_{l+1}+\epsilon_{l+1} R_{l}^{*}+\epsilon_{l}^{*} \epsilon_{l+1}\right) r d r
\end{array}
$$


and the absolute error is approximately

$$
2 \sum_{l=0}^{\infty} C_{l+1} \int_{0}^{\infty}\left(\epsilon_{l}^{*} R_{l+1}+R_{l}^{*} \epsilon_{l+1}\right) r d r .
$$

An interesting observation is that the algorithm for the bound polarization will not treat an identical perturbation in the same manner. The difference arises from the fact that the errors are distributed to all projection coefficients with the same $l$ values as the $R_{l}$ state

$$
\begin{aligned}
\tilde{R}_{l} & =R_{l}+\epsilon_{l} \\
\tilde{a}_{n, l} & =a_{n, l}+\int_{0}^{\infty} S_{n, l} \epsilon_{l} d r .
\end{aligned}
$$

The reconstructed bound part of the solution $\rho_{l}(r)$ therefore includes the compounded error

$$
\begin{aligned}
\rho_{l} & =\sum_{n=0}^{\infty} \tilde{a}_{n, l} S_{n, l} \\
\rho_{l}(r) & =\sum_{n=0}^{\infty} a_{n, l} S_{n, l}(r)+\sum_{n=0}^{\infty}\left(\int_{0}^{\infty} S_{n, l} \epsilon_{l} d r\right) S_{n, l}(r) .
\end{aligned}
$$

Defining a new coefficient

$$
\alpha_{l}(r) \equiv \sum_{n=0}^{\infty}\left(\int_{0}^{\infty} \epsilon_{l} S_{n, l} d r\right) S_{n, l}(r)
$$

then

$$
\begin{array}{r}
\tilde{P}_{b}=2 \sum_{l=0}^{\infty} C_{l+1} \int_{0}^{\infty} \Re\left(\tilde{\rho}_{l+1}^{*} \tilde{\rho}_{l}\right) r d r \\
\tilde{P}_{b}=P_{b}+\sum_{l=0}^{\infty} C_{l+1} \int_{0}^{\infty}\left(\alpha_{l}^{*} \rho_{l+1}+\rho_{l}^{*} \alpha_{l+1}\right) r d r+O\left(\alpha^{2}\right) .
\end{array}
$$

The final result of this analysis is that, while the form for the errors of $P(t)$ and $P_{b}(t)$ are the same, the coefficients are different: $\epsilon_{l}(r)$ versus

$$
\alpha_{l}(r)=\sum_{n=0}^{\infty}\left(\int_{0}^{\infty} S_{n, l} \epsilon_{l} d r\right) S_{n, l}(r) .
$$

The change in the value for the polarization due to this difference in the coefficients is seen in the results from section (4.1).

In contrast, an implicit method will alter the phase of the computed solution but not the amplitude

$$
R_{l} \rightarrow R_{l} e^{i \eta}
$$

Performing an identical analysis for a constant phase shift reveals that the errors to $P(t)$ and $P_{b}(t)$ are less dramatic and thus presumably also for a radially dependent phase shift. 
Considering the instantaneous susceptibility, $\Delta \alpha(I)$, it is determined from a linear extrapolation

$$
\lim _{Q \rightarrow 0} \chi=\chi^{(1)}+\Delta \alpha(I)
$$

Extrapolation is a notoriously sensitive numerical procedure [33] and thus one should also provide the relevant statistical measures of the accuracy of the value obtained. Therefore, we calculate the correlation coefficient

$$
c=\frac{\sigma_{x y}}{\sigma_{x x} \sigma_{y y}}
$$

and the standard error of the extrapolated y-intercept $(b)$

$$
S E=\sqrt{\frac{\sigma_{y y}-b \sigma_{x y}}{\sigma_{x x}(N-2)}} .
$$

In these equations $\bar{x}$ and $\bar{y}$ are the mean values of the $N$ data points and

$$
\sigma_{x y}=\sum_{n}\left(x_{n}-\bar{x}\right)\left(y_{n}-\bar{y}\right) .
$$

An absolute value of the correlation coefficient close to unity indicates a strong linear relationship between $y$ and $x$. To perform the extrapolation, we use the regression function from XMGRACE [42].

\section{Numerical implementation}

The accurate numerical solution of the equation

$$
\frac{\partial R_{l}}{\partial t}=\frac{i}{2} \frac{\partial^{2} R_{l}}{\partial r^{2}}-i \tilde{V}_{l}(r) R_{l}+i r E(t)\left(C_{l+1} R_{l+1}+C_{l-1} R_{l-1}\right)
$$

from our previous analysis requires careful consideration. First, the equation is defined on the semi-infinite domain $[0, \infty)$ with the boundary condition $R_{l}(0)=0$ for all $l$ states. A numerical algorithm must enforce this boundary and be reasonably accurate in space in order to allow for an as large as possible computational domain $[0, L]$. Second, the solution to the Schrödinger equation is conservative and amplitude errors can have a large effect on the computation of the polarization. The numerical propagator should thus also conserve the norm of the uncoupled $R_{l}$ states. Third, although the initial condition for all simulations is the atom in its ground state $\phi_{n=1, l=0}$, the coupling coefficients will excite states of arbitrarily high $l$ number. No numerical scheme can in practice be fully consistent with this requirement but must allow for convergence of the results as the number of $R_{l}$ states increases.

Considering these requirements, we have chosen a Strang-split second-order time stepping with multidomain pseudospectral derivatives. In the interests of clarity and reproducibility, we provide the following details.

3.1. Time stepping. Equation (3.1) involves a coupling in space through the second derivative and a coupling across momentum states. It is numerically advantageous to Strang split these two actions from each other.

Step $1(\delta T / 2) \frac{\partial R_{l}}{\partial t}=\operatorname{ir} E(t)\left(C_{l+1} R_{l+1}+C_{l-1} R_{l-1}\right)$

Step $2(\delta T) \frac{\partial R_{l}}{\partial t}=\frac{i}{2} \frac{\partial^{2} R_{l}}{\partial r^{2}}-i \tilde{V}_{l}(r) R_{l}$ 
Step $3(\delta T / 2) \frac{\partial R_{l}}{\partial t}=\operatorname{ir} E(t)\left(C_{l+1} R_{l+1}+C_{l-1} R_{l-1}\right)$

Numerically solving the full system of equations without this splitting is computationally demanding as the matrix involved would require continual updates in time. Obviously each step of the Strang splitting must be performed for each $l$ state before proceeding to the next.

There are two principle advantages to this splitting. First is that it is conservative. Secondly, the coupling of the momentum states can be performed very efficiently. Consider the corresponding matrix equation for a fixed $r$

$$
\frac{\partial \vec{R}}{\partial t}=i E r \bar{C} \vec{R}
$$

The coupling matrix $\bar{C}$ is a Hermitian matrix and thus has a Schur factorization $\bar{C}=\bar{Q} \bar{D} \bar{Q}^{H}$ which need only be computed once. More importantly, the matrix $\bar{D}$ is a diagonal matrix of eigenvalues. The solution to the matrix equation with the factorization is then

$$
\vec{R}(t+\delta t)=Q e^{i r \bar{D} \int_{t}^{t+\delta t} E(\tau) d \tau} Q^{*} \vec{R}(t) .
$$

The pulse shape $E(t)$ is known and thus the integral in the propagator could be computed analytically. However, since Strang splitting will add a second order error term, we opt for an approximation of the integral using Simpson's rule

$$
\int_{t}^{t+\delta t / 2} E(\tau) d \tau \approx \frac{\delta t}{2}\left(\frac{E(t)}{6}+\frac{2 E(t+\delta t / 4)}{3}+\frac{E(t+\delta t / 2)}{6}\right) .
$$

The more problematic term for a time-stepping algorithm is the effective potential which becomes large as $r \rightarrow 0$. The result is an imaginary eigenvalue which grows as the spacial grid is refined and which, for an explicit method, must be scaled by the time step $\delta t$ to bring the product into the domain of stability. An implicit solver which does not suffer from the severe stability requirement imposed by the potential near the origin is the implicit Crank-Nicolson method. Although it requires the inversion of a matrix, it has been found to be more efficient and accurate than other explicit solvers.

Finally, to improve the speed of the simulations we do not update the solution using the middle step of the time splitting at spatial points where it falls below a given tolerance, typically the machine precision $\left(\approx 10^{-16}\right)$ [31]. For positions where the solution falls below this value the derivative is assumed to be zero. This is justified by the fact that the solution decays dramatically as $r \rightarrow \infty$. The savings in simulation time of using this approximation is substantial.

3.2. Spatial derivatives. The $R_{l}(r)$ radial solutions contain contributions from both the bound atomic states and the high energy ionized free electron states. Due to this wide variation in energy, the $R_{l}$ states are potentially highly oscillatory. A numerical method for the spatial derivatives of these functions must therefore be capable of resolving these oscillations. Since the computational domains are potentially large, the numerical algorithm should also be accurate and require as little memory as possible. For the smooth solutions to the Schrödinger equation, a pseudospectral method has these qualities [18, 37, 40].

In our particular case, we have opted for a multidomain Legendre polynomial approach $[11,22,38]$. The computational grid consists of joined cells of length two. 
The non-uniformly distributed points within each cell are the nodes of the Legendre polynomial basis. The pseudospectral differentiation matrix is applied to the interior points and a high-order finite difference stencil updates the boundaries. To define the second derivative matrix, we take the product of two first derivative matrices. We have found that this approach provides more than sufficient accuracy.

There are three principle advantages to using a Legendre polynomial basis. The first advantage is that they are naturally defined on a finite interval $[0,2]$. They therefore can be easily spliced together to cover the entire semi-infinite interval. In contrast, the more common Fourier-Sine, Laguerre, or rational Chebyshev methods involve an arbitrary scaling of the domain. Furthermore, these methods are sensitive to the scaling parameter and thus require a complicated analysis to determine a near-optimal value $[3,5,25,39]$. Also, although no simple formula for the Legendre polynomials weights and nodes exist, they can be easily determined numerically [11].

A second motivation for choosing the Legendre polynomials as a basis is their relationship to Gaussian quadrature [36]. To compute the spatial integral over the entire computational domain, we use Gauss-Legendre-Lobatto quadrature on each closed element and sum the results. The Gauss-type quadrature rule $\int_{a}^{b} w(x) f(x) d x \approx$ $\sum_{k} W_{k} f\left(x_{k}\right)$ with this basis has weight $w(x)=1$ and the coefficients $W_{k}$ are known to high precision [13]. The integral of the continuous $R_{l}(r)$ functions can thus be computed in a straightforward manner.

The third advantage of the multidomain Legendre basis approach is the bandedness of the resulting differentiation matrix for the entire domain. Although the pseudospectral differentiation matrix for each cell is dense, by applying a multidomain approach, one can obtain a block-diagonal matrix for the entire domain. This is particularly important for an efficient implementation of the Crank-Nicolson solver which involves an inversion of this matrix. The block-diagonal differentiation matrix is easily inverted using a standard complex valued banded matrix solver such as the LAPACK function $z g b s v$ [1]. The actual structure of the full matrix will of course depend however on the choice of algorithm for the boundary points. We therefore now turn to this issue.

To update the boundary point shared by two neighboring cells, we utilize a 9-point finite difference stencil. Since the grid is not uniform, the coefficients of the stencil are nontrivial but can be easily derived [17]. As for the choice of a Legendre basis for the interior points, there are three motivations for this approach to the boundaries.

First and foremost is consistency. Although one could in principle solve the equations on the interior and then enforce continuity of the solution and its derivatives to a finite order, it has been found that this leads to artificial cusps and inconsistencies near the boundary points [21]. The finite-difference stencil naturally allows information to pass across the boundary between cells.

Secondly, the symmetric stencil maintains the conservative nature of the differentiation matrix. The common approach from fluid dynamics of applying a penalty at the boundaries leads to artificial damping. Furthermore, we have found that these ad hoc boundary schemes do not preserve the smoothness of the solution over long time simulations.

Thirdly, since the distance between the boundary point and its nearest neighbor goes as $1 / N^{2}$ where $N$ is the degree of the polynomial basis, the approximate derivative obtained from the stencil is quite good. Using 16 points per cell, a test of the 9-point stencil reveals that the errors are of the same magnitude as those incurred by our second derivative pseudospectral matrix. 
Finally, since the computational domain is finite, the solution must be damped at the end in order to avoid unphysical reflections from the $R_{l}\left(r_{\max }\right)=0$ boundary condition. We have chosen a filter of the form

$$
\alpha(r)= \begin{cases}1 & r \leq\left(r_{\max }-4\right) \\ 1-e^{\left(r-\left(r_{\max }-4\right)\right)^{-4}} & \text { else }\end{cases}
$$

This is different from the one utilized in [31] but performs well. The boundary condition $R_{l}(r=0)=0$ is explicitly enforced.

\section{Results}

For all simulations in this section, unless otherwise stated, we utilize the parameters in Table 4.1. The angular frequency $\omega$ and period $\tau$ in atomic units corresponding

TABLE 4.1. Simulation Parameters

\begin{tabular}{cc}
\hline Wavelength $(\mathrm{nm})$ & 800 \\
Time steps per cycle & 1024 \\
Domain length $(\mathrm{au})$ & 3200 \\
$R_{l}$ states & 35 \\
Nodes per cell & 8 \\
Interface stencil width (points) & 9 \\
Bound states $\left(\phi_{n, l}\right) n_{\max }$ & 39 \\
\hline
\end{tabular}

to this wavelength are approximately 0.0569538 and 110.321 , respectively.

To excite the atom, we have considered two profiles for the electric field. The first is the sine-square ramped electric field profile utilized by Nurhuda [31], shown in Fig. 4.1. The equation for this profile is

$$
E(t)=\sin (2 \pi t / \tau) \begin{cases}E_{\text {max }} \cdot \sin ^{2}\left(\frac{\pi t}{2 \cdot T_{r}}\right) & t \leq T_{r} \\ E_{\text {max }} & \text { else }\end{cases}
$$

where the ramp time $T_{r}$ is set to $3 \tau$. The other pulse we have studied is Gaussian and shown in Fig. 4.2. This excitation is given by the equation

$$
\begin{aligned}
E(t) & =E_{\max } \sin (2 \pi t / \tau) \exp \left[-0.5 \cdot\left(\frac{\left(t-0.5 \cdot\left(T_{r}+T_{p}\right)-0.25 \cdot \tau\right)}{\sigma \cdot T_{p}}\right)^{2}\right], \\
T_{r} & =2 \tau \\
T_{p} & =4 \tau \\
\sigma & =1 / 5 .
\end{aligned}
$$

In order to accurately determine the temporal Fourier transform of the polarization and electric field, we wait a time of $5 \tau$ in addition to the $6 \tau$ from the Gaussian pulse. The full time history of both pulses is thus $11 \tau$.

For previous studies of high-harmonic generation by multiphoton ionization $[19,24]$, the sine-square pulse has had the advantage of creating a large amount of ionization. However, for the purposes of computing the transform of the polarization and electric fields, the Gaussian pulse has the distinct advantage of possessing a unique and well-defined Fourier transform. For these reasons, the transforms computed for the sine-square pulse do not include the initial ramp while those for the Gaussian include the full time history. 


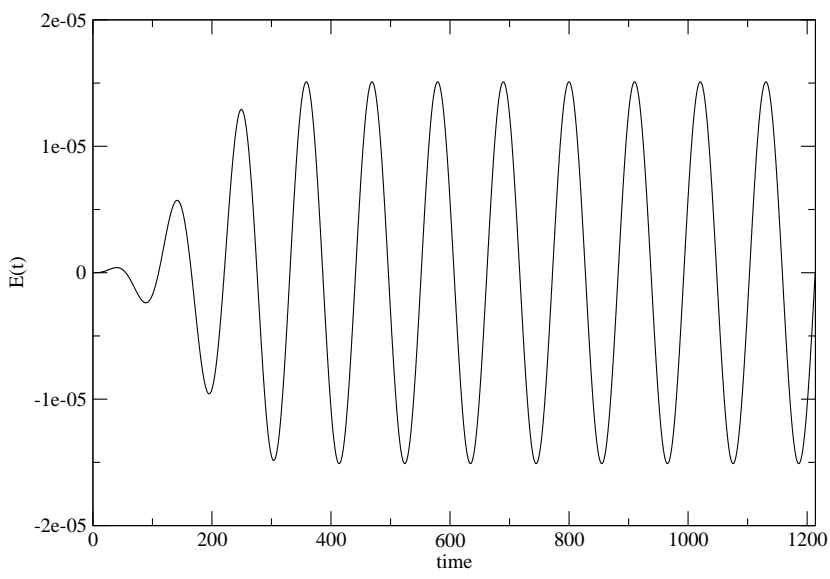

Fig. 4.1. Sine-Square Pulse Profile, $I=8 \cdot 10^{6}\left(w / \mathrm{cm}^{2}\right)$



Fig. 4.2. Gaussian Pulse Profile, $I=8 \cdot 10^{6}\left(w / \mathrm{cm}^{2}\right)$

4.1. Numerical sensitivity analysis. A revealing test of our approach is a comparison between the numerically determined values for the linear susceptibility $\chi_{b}^{(1)}(\omega)$ and those obtained from the analytic expression (2.23) in section (2.2). Table 4.2 compares the values for different $\omega$ computed with the two pulses and a Mathematica script. As expected, the values for $\chi_{b}^{(1)}(\omega)$ are independent of the shape of the pulse. Fitting the values of $\chi_{b}^{(1)}(\omega)$ by a quintic polynomial and extrapolating to $\omega=0$ yields a value of 3.6701 for the sine-square pulse and 3.6703 for the Gaussian. This is close to the exact value 3.6629 obtained from equation (2.23).

A similar analysis can be made for the total linear susceptibility $\chi^{(1)}(\omega)$. However, the analytic expression is more difficult to compute as it involves an integration over all continuum states. Thus, we have determined $\chi^{(1)}(\omega=0)$ from fitting the data in Table 4.3 and again extrapolating with a quintic polynomial to $\omega=0$. This yields an estimate for the static polarizability from the sine-square pulse of 4.5084 and the Gaussian pulse 4.5054, in close agreement with the exact value 4.5. 
TABLE 4.2. $\Re\left(\chi_{b}^{(1)}\left(\omega, T_{\text {final }}\right)\right) I=1 \cdot 10^{4}\left(w / \mathrm{cm}^{2}\right)$

\begin{tabular}{ccccc}
\hline \hline$\lambda(n m)$ & $\omega(a . u)$. & Exact & Sine-Square & Gaussian Pulse \\
\hline 200 & 0.227816 & 5.6258 & 5.6293063 & 5.6085268 \\
400 & 0.113908 & 4.0103 & 4.0085727 & 4.0085195 \\
600 & 0.075938 & 3.8096 & 3.8080204 & 3.8080155 \\
800 & 0.056953 & 3.7441 & 3.7427883 & 3.7427877 \\
1000 & 0.045563 & 3.7145 & 3.7135762 & 3.7135761 \\
1200 & 0.037969 & 3.6987 & 3.6981273 & 3.6981273 \\
\hline
\end{tabular}

TABLE 4.3. $\Re\left(\chi^{(1)}\left(\omega, T_{\text {final }}\right)\right) I=1 \cdot 10^{4}\left(w / \mathrm{cm}^{2}\right)$

\begin{tabular}{cccc}
\hline \hline$\lambda(n m)$ & $\omega($ a.u. $)$ & Sine-Square & Gaussian Pulse \\
\hline 200 & 0.227816 & 6.6031465 & 6.5827767 \\
400 & 0.113908 & 4.8769182 & 4.8768649 \\
600 & 0.075938 & 4.6596395 & 4.6595346 \\
800 & 0.056953 & 4.5885195 & 4.5885189 \\
1000 & 0.045563 & 4.5565801 & 4.5565800 \\
1200 & 0.037969 & 4.5395751 & 4.5395751 \\
\hline
\end{tabular}

To test convergence, we have increased the number of steps per oscillation and studied the effect on $\chi_{b}$ with the full time history for the Gaussian pulse. The results are given in Table 4.1. There are a couple of conclusions which can be drawn from this data.

TABle 4.4. $\chi_{b}$ and Relative Error vs Time Steps per Cycle, $I=8 \cdot 10^{6}\left(w / \mathrm{cm}^{2}\right), \quad$ Gaussian Pulse, Exact 3.7441, Analytic for 39 bound states 3.7421 .

\begin{tabular}{cccc}
\hline \hline$N$ & $\chi_{b}$ & Exact R.E. $(\%)$ & $\chi_{b}^{(1)}(39)$ R.E. $(\%)$ \\
\hline 128 & $3.7837+2.8867 \cdot 10^{-6} i$ & 1.0578 & 1.1118 \\
256 & $3.7525+2.7126 \cdot 10^{-6} i$ & 0.2249 & 0.2785 \\
512 & $3.7447-9.9198 \cdot 10^{-7} i$ & 0.01693 & 0.0704 \\
1024 & $3.7428-1.9667 \cdot 10^{-6} i$ & 0.03505 & 0.01838 \\
2048 & $3.7423-2.1590 \cdot 10^{-6} i$ & 0.04804 & 0.005380 \\
\hline
\end{tabular}

First, that the susceptibility converges to an incorrect value reveals the inconsistency of computing this quantity from only a fraction of the bound states. The value is however the one obtained from the analytic formula for a finite number of bound states. Increasing the number of bound states to improve the computed susceptibility is possible but requires a dramatically larger domain as the radial position of the maximum of the shell amplitude $S_{n, l}$ is proportional to $n^{2}$.

Second, the fact that the relative error of the real part of the computed susceptibility decreases by almost exactly a factor of 4 as the number of time steps is doubled, 
suggests that the majority of the simulation error is due to the second order $(\delta t)^{2}$ time stepping.

Third, for low intensities, where little ionization occurs, the imaginary part of the computed susceptibility should be close to zero. This is observed. However, the very small size of the imaginary part brings it close to the limit of the accuracy of the simulations. Any conclusions based on this value therefore should be made with caution.

TABle 4.5. Additive Noise: Projection Coefficient Magnitudes

\begin{tabular}{ccccc}
\hline \hline$n$ & $l$ & noise $=0$ & noise $=10^{-8}$ & noise $=10^{-4}$ \\
\hline 1 & 0 & 0.990000000763 & 0.990000008747 & 0.990079850612 \\
2 & 0 & $6.6674 \cdot 10^{-11}$ & $2.1306 \cdot 10^{-8}$ & $2.1344 \cdot 10^{-4}$ \\
2 & 1 & 0.01 & 0.01000001795 & 0.01018 \\
3 & 0 & $2.4918 \cdot 10^{-11}$ & $2.7322 \cdot 10^{-8}$ & $2.7305 \cdot 10^{-4}$ \\
3 & 1 & $2.2422 \cdot 10^{-15}$ & $2.4180 \cdot 10^{-8}$ & $2.4180 \cdot 10^{-4}$ \\
3 & 2 & 0 & 0 & 0 \\
4 & 0 & $1.3961 \cdot 10^{-11}$ & $2.8309 \cdot 10^{-8}$ & $2.8318 \cdot 10^{-4}$ \\
4 & 1 & $1.1837 \cdot 10^{-15}$ & $2.9732 \cdot 10^{-8}$ & $2.9732 \cdot 10^{-4}$ \\
4 & 2 & 0 & 0 & 0 \\
4 & 3 & 0 & 0 & 0 \\
\hline
\end{tabular}

TABle 4.6. Additive Noise: Polarization Sensitivity $(n=2, l=1)$

\begin{tabular}{cccccc}
\hline \hline Noise Level & 0 & $10^{-6}$ & $10^{-5}$ & $10^{-4}$ & $10^{-3}$ \\
\hline Full & 0.0147497236748 & 0.014755259 & 0.01506729 & 0.044146 & 2.9308 \\
Bound & 0.0147497236826 & 0.014754703 & 0.01503925 & 0.041618 & 2.6808 \\
\hline
\end{tabular}

TABle 4.7. Additive Noise: Polarization Sensitivity $(n=15, l=1)$

\begin{tabular}{ccccc}
\hline \hline Noise Level & 0 & $10^{-6}$ & $10^{-5}$ & $10^{-4}$ \\
\hline Full & 0.000429580641121 & 0.0004147664 & 0.00054365 & 0.027791 \\
Bound & 0.000429580640952 & 0.0004142325 & 0.00051583 & 0.025265 \\
\hline
\end{tabular}

Finally, considering the analysis of the sensitivity of the polarization to noise in section (2.4), we have chosen to investigate this numerically. In particular, we have computed the polarization corresponding to a coupling between the ground state $R_{0}=0.99 \cdot S_{1,0}$ and an excited state $R_{l}=0.01 \cdot S_{n, l}$. We have then perturbed these states with a uniformly distributed random variable $\eta$ with fixed maximum amplitude.

$$
R_{l}(r) \rightarrow R_{l}(r)+\eta(r)
$$

The effect of these perturbations on the projection coefficients is shown in Table 4.5. 
The errors in the projection coefficients for the case without noise is due primarily to the Gaussian quadrature. Table 4.6 and 4.7 reveal that the effect is more significant for larger principle quantum numbers.

As expected from our previous analysis, the two algorithms for the total and bound polarization respond differently to the additive noise. Furthermore, repeating the same experiment with a random noise to the phase of the shell amplitudes reveals that the computations are far less sensitive, see Table 4.8 and 4.9.

TABle 4.8. Multiplicative Noise: Polarization Sensitivity ( $n=2, l=1$ )

\begin{tabular}{cccc}
\hline \hline Noise Level & 0 & $10^{-3}$ & $10^{-2}$ \\
\hline Full & 0.0147497236748 & 0.014749722797 & 0.01474963588 \\
Bound & 0.0147497236826 & 0.014749723008 & 0.01474965626 \\
\hline
\end{tabular}

TABLE 4.9. Multiplicative Noise: Polarization Sensitivity $(n=15, l=1)$

\begin{tabular}{cccc}
\hline \hline Noise Level & 0 & $10^{-3}$ & $10^{-2}$ \\
\hline Full & 0.000429580641121 & 0.0004295806185 & 0.00042957838 \\
Bound & 0.000429580640952 & 0.0004295807258 & 0.00042958913 \\
\hline
\end{tabular}

These results confirm our choice of the conservative Crank-Nicolson solver and highlight the necessity of highly accurate spatial integration and derivatives.

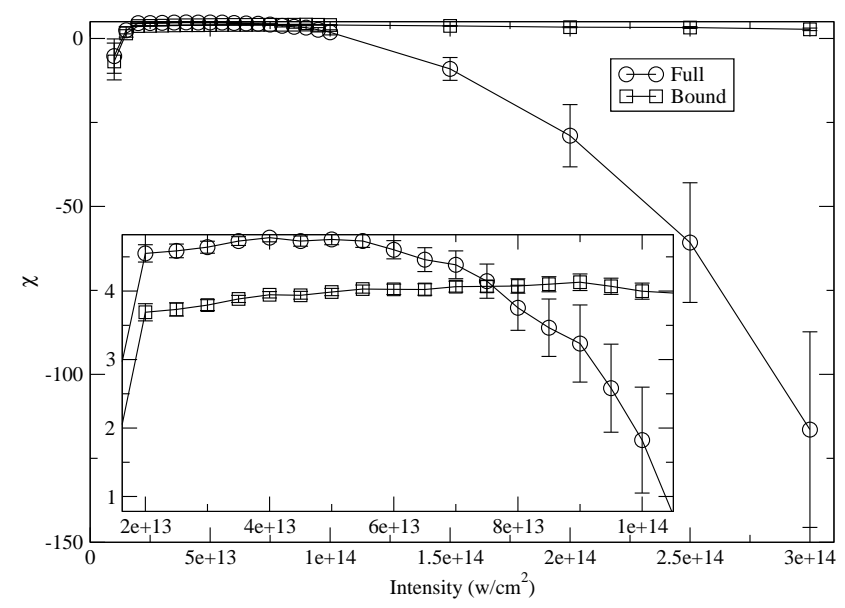

FIG. 4.3. Sine-Square Pulse: Instantaneous $\chi$ versus $I\left(w / \mathrm{cm}^{2}\right)$

4.2. Sine-square pulse results. With the theory established and the convergence of the code confirmed, it is now possible to report our numerical findings. In Fig. 4.3 is the instantaneous portion of the susceptibility from the sine-square pulse. To be specific about the plotted values and thus make the results easier to reproduce, we include them in Table 4.10 and 4.11. 
TABle 4.10. Sine-Square Pulse: Instantaneous $\chi$ versus $I\left(w / \mathrm{cm}^{2}\right)$, 3 Cycles Deleted

\begin{tabular}{ccccc}
\hline \hline$I\left(w / \mathrm{cm}^{2}\right)$ & $\chi\left(T_{\text {final }}\right)$ & $\chi$ Instant & Correlation & Standard Error \\
\hline $1 \cdot 10^{2}$ & 4.5885 & 4.4454 & -0.2978 & 0.1873 \\
$1 \cdot 10^{4}$ & 4.5885 & 4.4584 & -0.2516 & 0.2043 \\
$1 \cdot 10^{6}$ & 4.5885 & 4.4546 & -0.2623 & 0.2011 \\
$1 \cdot 10^{8}$ & 4.5885 & 4.5054 & -0.2771 & 0.1177 \\
$1 \cdot 10^{10}$ & 4.5886 & 10.3971 & -0.2879 & 7.8867 \\
$1 \cdot 10^{12}$ & 4.5940 & -57.2130 & 0.1704 & 145.8835 \\
$1 \cdot 10^{13}$ & 4.6451 & -5.2503 & 0.6192 & 5.1215 \\
$1.5 \cdot 10^{13}$ & 4.6752 & 2.4380 & 0.6479 & 1.0721 \\
$2 \cdot 10^{13}$ & 4.7033 & 4.5502 & 0.4348 & 0.1268 \\
$2.5 \cdot 10^{13}$ & 4.7329 & 4.5866 & 0.5007 & 0.1000 \\
$3 \cdot 10^{13}$ & 4.7538 & 4.6407 & 0.4596 & 0.08744 \\
$3.5 \cdot 10^{13}$ & 4.7352 & 4.7305 & 0.1456 & 0.06410 \\
$4 \cdot 10^{13}$ & 4.6490 & 4.7800 & -0.3601 & 0.06095 \\
$4.5 \cdot 10^{13}$ & 4.6664 & 4.7298 & -0.06633 & 0.07449 \\
$5 \cdot 10^{13}$ & 4.4106 & 4.7538 & -0.6778 & 0.07729 \\
$5.5 \cdot 10^{13}$ & 4.0019 & 4.7313 & -0.8564 & 0.09376 \\
$6 \cdot 10^{13}$ & 3.9452 & 4.6034 & -0.7218 & 0.1331 \\
$6.5 \cdot 10^{13}$ & 3.5685 & 4.4587 & -0.7238 & 0.1741 \\
$7 \cdot 10^{13}$ & 1.9543 & 4.3839 & -0.9356 & 0.2029 \\
$7.5 \cdot 10^{13}$ & 0.9708 & 4.1443 & -0.9443 & 0.2499 \\
$8 \cdot 10^{13}$ & 1.2228 & 3.7560 & -0.8635 & 0.3293 \\
$8.5 \cdot 10^{13}$ & -0.3011 & 3.4648 & -0.8926 & 0.4196 \\
$9 \cdot 10^{13}$ & -3.3581 & 3.2339 & -0.9384 & 0.5627 \\
$9.5 \cdot 10^{13}$ & -5.4926 & 2.5820 & -0.9440 & 0.6428 \\
$1 \cdot 10^{14}$ & -6.2603 & 1.8236 & -0.9233 & 0.7730 \\
$1.5 \cdot 10^{14}$ & -48.8051 & -9.0673 & -0.9438 & 3.4011 \\
$2 \cdot 10^{14}$ & -117.5356 & -28.9546 & -0.9250 & 9.2575 \\
$2.5 \cdot 10^{14}$ & -158.5233 & -60.7687 & -0.8273 & 17.8161 \\
$3 \cdot 10^{14}$ & -178.8479 & -116.4518 & -0.4841 & 29.1142 \\
\hline & & & &
\end{tabular}

The instantaneous values are obtained by taking the limit of the susceptibility averaged over a carrier cycle with respect to the averaged ionization probability.

$$
\begin{array}{r}
\chi^{(1)}+\Delta \alpha(I)=\lim _{\bar{Q}_{c} \rightarrow 0} \bar{\chi}_{c} \\
\bar{Q}_{c}=\frac{1}{N} \sum_{n=0}^{N-1} Q(c \cdot \tau+n \delta t) \\
\bar{\chi}_{c}=\frac{1}{N} \sum_{n=0}^{N-1} \chi(c \cdot \tau+n \delta t)
\end{array}
$$


TABle 4.11. Sine-Square Pulse: Instantaneous $\chi_{b}$ versus $I\left(w / \mathrm{cm}^{2}\right)$, 3 Cycles Deleted

\begin{tabular}{ccccc}
\hline \hline$I\left(w / \mathrm{cm}^{2}\right)$ & $\chi_{b}\left(T_{\text {final }}\right)$ & $\chi_{b}$ Instant & Correlation & Standard Error \\
\hline $1 \cdot 10^{2}$ & 3.7427 & 3.5916 & -0.2981 & 0.1975 \\
$1 \cdot 10^{4}$ & 3.7427 & 3.6053 & -0.2520 & 0.2155 \\
$1 \cdot 10^{6}$ & 3.7427 & 3.6013 & -0.2627 & 0.2121 \\
$1 \cdot 10^{8}$ & 3.7427 & 3.6550 & -0.2775 & 0.1241 \\
$1 \cdot 10^{10}$ & 3.7428 & 9.8933 & -0.2883 & 8.3393 \\
$1 \cdot 10^{12}$ & 3.7487 & -63.7857 & 0.1705 & 159.3329 \\
$1 \cdot 10^{13}$ & 3.8038 & -6.8518 & 0.6217 & 5.4795 \\
$1.5 \cdot 10^{13}$ & 3.8363 & 1.4999 & 0.6531 & 1.1043 \\
$2 \cdot 10^{13}$ & 3.8698 & 3.6903 & 0.4941 & 0.1253 \\
$2.5 \cdot 10^{13}$ & 3.9072 & 3.7318 & 0.5799 & 0.09631 \\
$3 \cdot 10^{13}$ & 3.9428 & 3.7949 & 0.5823 & 0.07929 \\
$3.5 \cdot 10^{13}$ & 3.9691 & 3.8850 & 0.5200 & 0.05240 \\
$4 \cdot 10^{13}$ & 3.9752 & 3.9457 & 0.3320 & 0.04636 \\
$4.5 \cdot 10^{13}$ & 4.0377 & 3.9386 & 0.5526 & 0.05733 \\
$5 \cdot 10^{13}$ & 4.0602 & 3.9863 & 0.5166 & 0.05347 \\
$5.5 \cdot 10^{13}$ & 4.0556 & 4.0291 & 0.3673 & 0.05945 \\
$6 \cdot 10^{13}$ & 4.1157 & 4.0249 & 0.5015 & 0.07604 \\
$6.5 \cdot 10^{13}$ & 4.0889 & 4.0225 & 0.4454 & 0.08359 \\
$7 \cdot 10^{13}$ & 3.9121 & 4.0645 & -0.1155 & 0.07989 \\
$7.5 \cdot 10^{13}$ & 3.9878 & 4.0685 & 0.1860 & 0.08443 \\
$8 \cdot 10^{13}$ & 4.1202 & 4.0739 & 0.4792 & 0.1053 \\
$8.5 \cdot 10^{13}$ & 3.9651 & 4.0997 & 0.1525 & 0.1103 \\
$9 \cdot 10^{13}$ & 3.5650 & 4.1291 & -0.5736 & 0.1210 \\
$9.5 \cdot 10^{13}$ & 3.5494 & 4.0696 & -0.4925 & 0.1179 \\
$1 \cdot 10^{14}$ & 3.6934 & 3.9985 & 0.1166 & 0.1178 \\
$1.5 \cdot 10^{14}$ & 1.2398 & 3.7281 & -0.9529 & 0.1188 \\
$2 \cdot 10^{14}$ & -3.0858 & 3.3605 & -0.9917 & 0.1646 \\
$2.5 \cdot 10^{14}$ & -5.1076 & 3.2541 & -0.9895 & 0.2765 \\
$3 \cdot 10^{14}$ & -5.3155 & 2.7236 & -0.9828 & 0.3718 \\
\hline & & & &
\end{tabular}

The saturation of the full susceptibility is in agreement with the previous work by Nurhuda, et. al. [31] and as one can see from Table 4.11, the correlation coefficient has a magnitude near one for high intensities. This confirms the near linear dependence of the susceptibility on the ionization probability that one would expect from the Taylor series expansion (2.10). The wild behavior of the susceptibility for low intensities is a purely numerical artifact due to the fact that the ionization probability is near or below the accuracy of the simulations. The growth of the standard error as the intensity increases can also be easily explained. As the intensity of the incident light increases, so too does the ionization probability. Fitting a line to the $(Q, \chi)$ data for 
larger $Q$ values means that determining $\chi$ for $Q=0$ is an extrapolation over a larger $\Delta Q$ interval. Even small errors in a linear fit can thus be magnified by this distance into a considerable extrapolation error. The conclusion is thus that there exists only a window of ionization probability values for which the extrapolation procedure is robust.

More interesting is the correlation which exists between the ionization and $\chi_{b}$ for large intensities $\left(>10^{14}\left(\mathrm{w} / \mathrm{cm}^{2}\right)\right)$. While the low correlation coefficient for moderate intensities demonstrates an independence of $\chi_{b}$ from the ionization probability, the correlation at high intensities suggests that it is incorrect to attribute saturation solely to ionization. Diagrams which are more revealing of the ionization effects on $\chi$ and $\chi_{b}$ are in Fig.4.4 and Fig.4.5, which show the saturation of the susceptibility versus

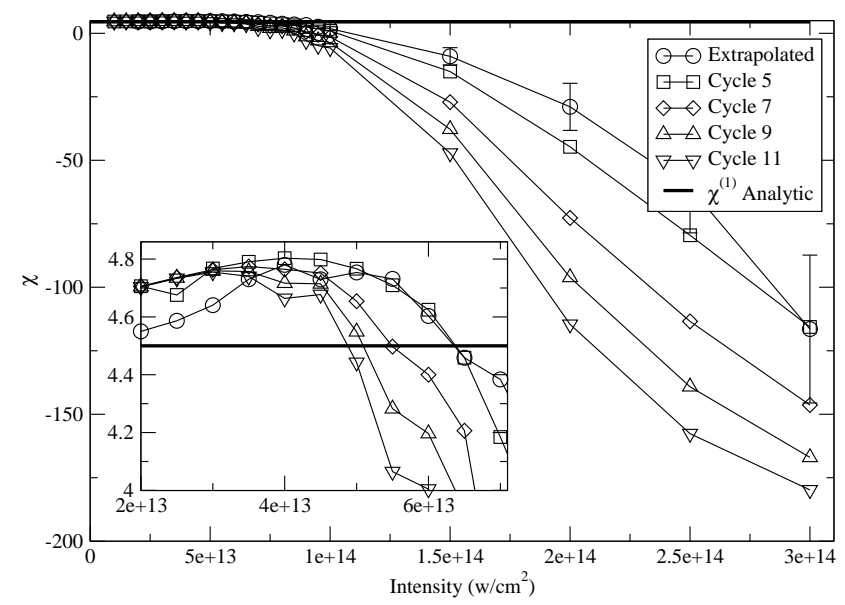

Fig. 4.4. Sine-Square Pulse: $\chi$ Averaged versus Cycle, Instant $\chi$ Cubic Polynomial Least Squares Fit, $\quad \chi(I)=4.6008+6.0116 .10^{-15} I+1.7899 .10^{-28} I^{2}-5.1205 .10^{-42} I^{3}$

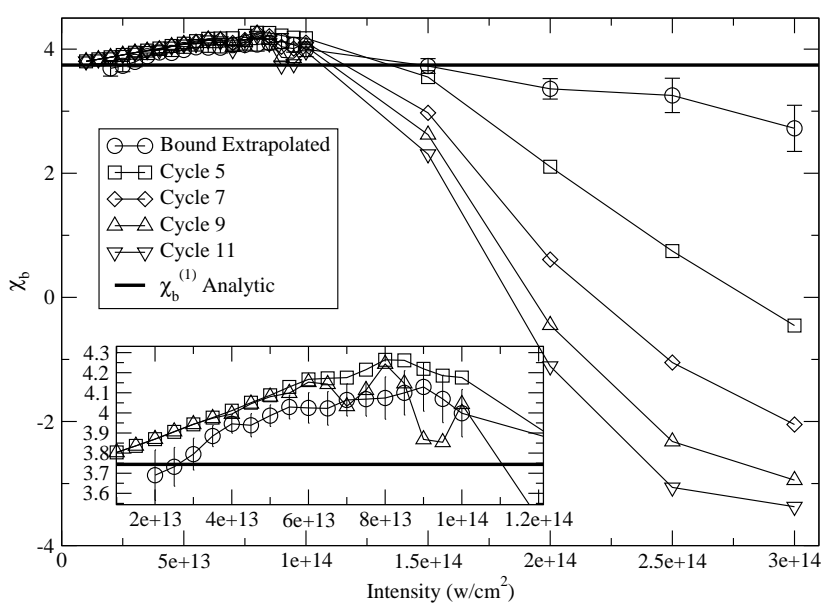

Fig. 4.5. Sine-Square Pulse: $\chi_{b}$ Averaged versus Cycle, Instant $\chi_{b}$ Cubic Polynomial Least Squares Fit, $\quad \chi_{b}(I)=3.4523+1.5709 \cdot 10^{-14} I-1.1587 \cdot 10^{-28} I^{2}+1.8727 \cdot 10^{-43} I^{3}$ 
TABLE 4.12. Sine-Square Pulse: Instant $\chi$ vs NRamp, $I=8 \cdot 10^{13}\left(w / \mathrm{cm}^{2}\right)$

\begin{tabular}{|c|c|c|c|c|c|}
\hline NRamp & $\overline{\overline{1}}$ & $\overline{2}$ & $\overline{3}$ & $\overline{44}$ & $\overline{5}$ \\
\hline \multicolumn{6}{|l|}{ Cycles Removed } \\
\hline \multicolumn{6}{|l|}{ NRamp } \\
\hline$\chi$ & 4.8133 & 4.1194 & 3.7560 & 3.5849 & 3.5384 \\
\hline Correlation & -0.9988 & -0.9398 & -0.86358 & -0.8621 & -0.8879 \\
\hline Std. Error & 0.03965 & 0.2309 & 0.3293 & 0.3369 & 0.3149 \\
\hline \multicolumn{6}{|l|}{ NRamp + 1} \\
\hline$\chi$ & 4.8632 & 4.6019 & 4.4423 & 4.2852 & 4.1913 \\
\hline Correlation & -0.998839 & -0.9996706 & -0.9999255 & -0.9999877 & -0.9999927 \\
\hline Std. Error & 0.04594 & 0.024032 & 0.01141 & 0.004725 & 0.003725 \\
\hline \multicolumn{6}{|l|}{ NRamp +2} \\
\hline$\chi$ & 4.7759 & 4.5553 & 4.4197 & 4.27897 & 4.1919 \\
\hline Correlation & -0.9997089 & -0.9999486 & -0.9999944 & -0.9999887 & -0.9999883 \\
\hline 0.02654 & 0.01113 & 0.003712 & 0.005376 & 0.005886 & \\
\hline
\end{tabular}

TABLE 4.13. Sine-Square Pulse: Instant $\chi_{b}$ vs NRamp, $I=8 \cdot 10^{13}\left(w / \mathrm{cm}^{2}\right)$

\begin{tabular}{cccccc}
\hline \hline NRamp & 1 & 2 & 3 & 4 & 5 \\
\hline Cycles Removed & & & & & \\
\hline NRamp & & & & & \\
\hline$\chi_{b}$ & 4.2359 & 4.1092 & 4.07392 & 4.0543 & 4.0414 \\
Correlation & 0.1767 & 0.4544 & 0.4792 & 0.4945 & 0.4976 \\
Std. Error & 0.05013 & 0.09307 & 0.1053 & 0.1068 & 0.1121 \\
\hline NRamp +1 & & & & & \\
\hline$\chi_{b}$ & 4.3414 & 4.3041 & 4.2932 & 4.2757 & 4.2732 \\
Correlation & -0.9958 & -0.9693 & -0.9374 & -0.8713 & -0.8489 \\
Std. Error & 0.002931 & 0.005861 & 0.007926 & 0.0105 & 0.01174 \\
\hline NRamp +2 & & & & & \\
\hline$\chi_{b}$ & 4.3377 & 4.3122 & 4.3059 & 4.2938 & 4.2938 \\
Correlation & -0.9953 & -0.9792 & -0.9735 & -0.9641 & -0.9652 \\
Std. Error & 0.003548 & 0.006492 & 0.007344 & 0.008385 & 0.008643 \\
\hline
\end{tabular}

time.

Finally, we have analyzed the effect of the number of ramp cycles on the values for $\chi$ and $\chi_{b}$. The results are presented in Table 4.12 and 4.13. What is interesting to note from these tables is the sensitivity to the shape of the pulse. While the correlation coefficient is always near one and thus clearly indicates a linear dependence on the ionization probability, the ramp up of the pulse persists in the history of the polarization even when the amplitude has been fixed to a constant value. Even more surprising, is the fact that $\chi_{b}$ has a strongly linear dependence if a sufficient number of initial cycles are eliminated from the extrapolation procedure. 
Turning to the time evolution of the polarization itself, Fig. 4.6 and 4.7, we find that the total and bound polarizations follow the electric field for low intensities. However, the complex behavior for large intensities makes a qualitative analysis difficult and the evaluation of the transform from a finite time history more difficult to justify. In the next section, we have therefore considered a Gaussian pulse, which exhibits a more simply interpreted behavior.

4.3. Gaussian pulse results. In order to ascertain if the results for the sine-square pulse are specific to that excitation, we repeat the simulations with the same parameters for the Gaussian pulse. From Fig. 4.8 and 4.9, which depict the total and bound polarizations, a clearer picture of the atom's response emerges. In particular, we find that for high intensities, since the polarization is proportional to the expected position of the electron and the electron may be ionized and free to move away from the nucleus, the total polarization grows linearly. In contrast, the bound

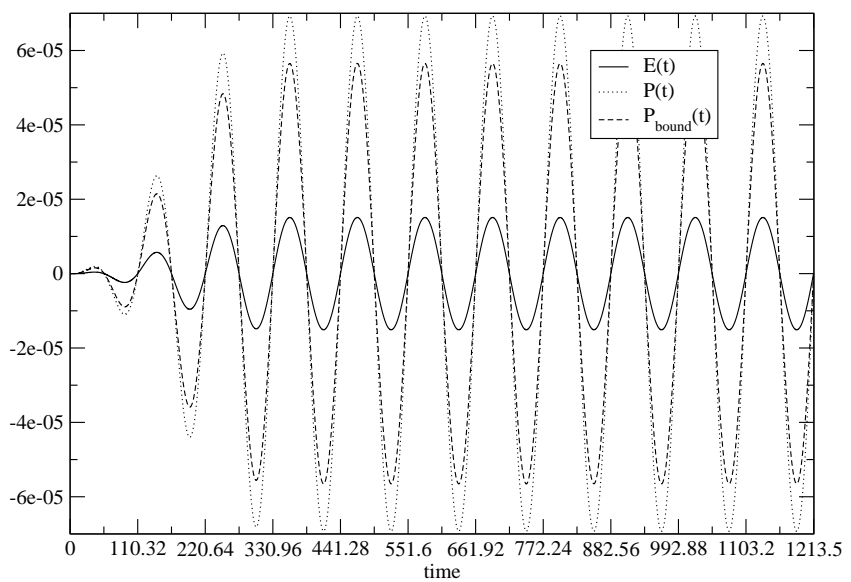

FIG. 4.6. Sine-Square Pulse: Polarization versus Time, $I=8 \cdot 10^{6}\left(\mathrm{w} / \mathrm{cm}^{2}\right)$

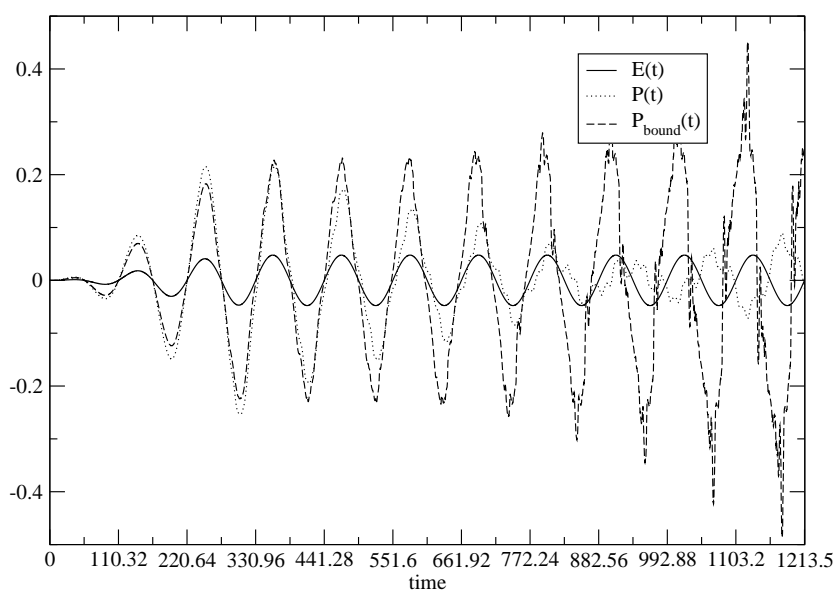

FIG. 4.7. Sine-Square Pulse: Polarization versus Time, $I=8 \cdot 10^{13}\left(\mathrm{w} / \mathrm{cm}^{2}\right)$ 


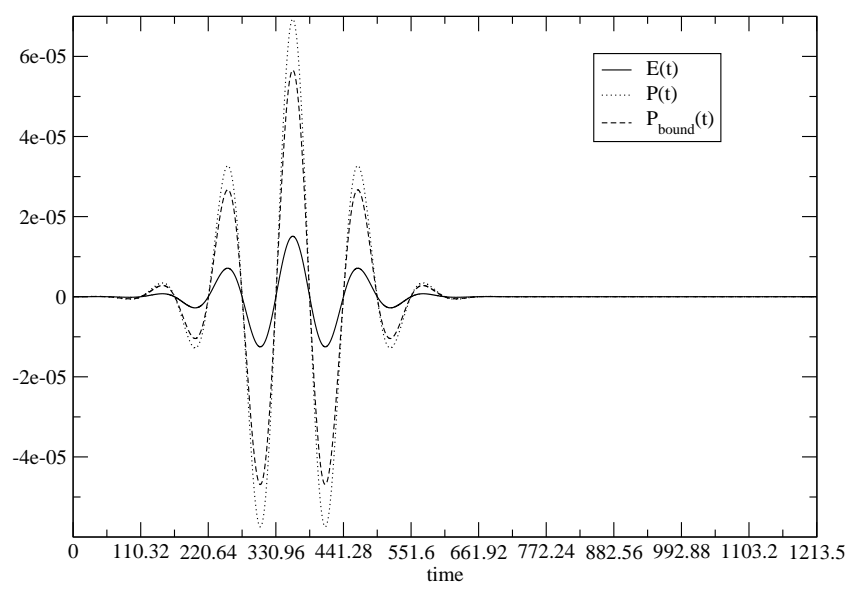

FIG. 4.8. Gaussian Pulse: Polarization versus Time, $I=8 \cdot 10^{6}\left(\mathrm{w} / \mathrm{cm}^{2}\right)$

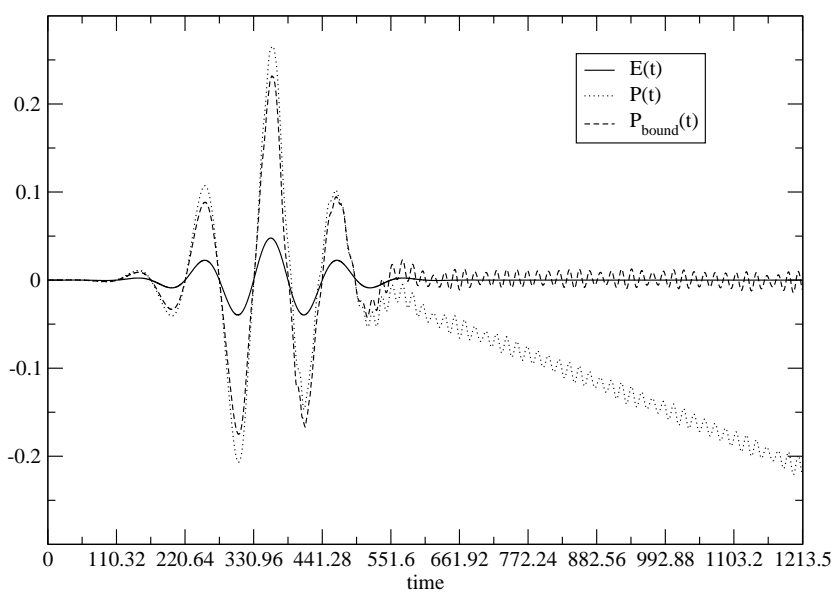

Fig. 4.9. Gaussian Pulse: Polarization versus Time, $I=8 \cdot 10^{13}\left(w / \mathrm{cm}^{2}\right)$

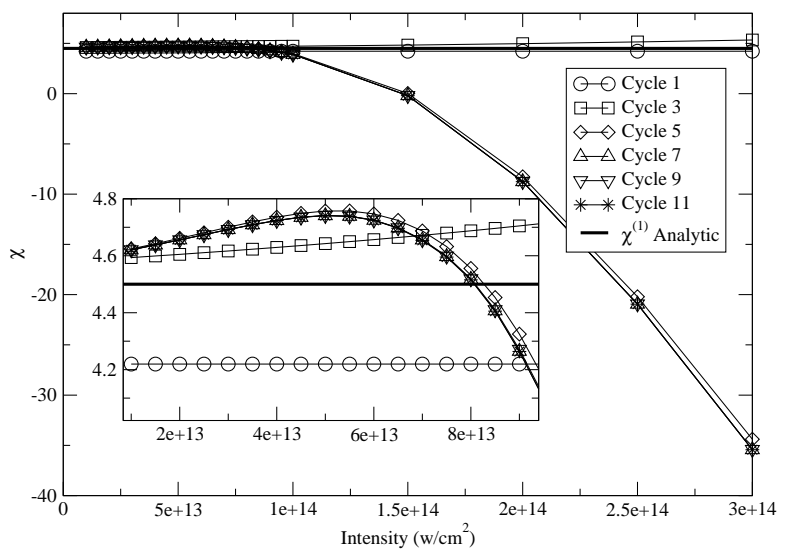

FIG. 4.10. Gaussian Pulse: $\chi$ versus Cycle 




Fig. 4.11. Gaussian Pulse: $\chi_{b}$ versus Cycle



FIG. 4.12. Gaussian Pulse: $\chi(t)$ vs $Q(t)$ for Pulses of Varying Width, $I=8 \cdot 10^{13}\left(\mathrm{w} / \mathrm{cm}^{2}\right)$

polarization oscillates around a value near zero.

With respect to the saturation of the susceptibility, this behavior is also observed for the Gaussian pulse in Fig. 4.10 and 4.11. Since the bound part of the susceptibility saturates as a function of intensity, we can conclude that the effect is not particular to the sine-square pulse and not solely due to the induced ionization.

Where the Gaussian pulse and sine-square pulse differ is in the long term behavior of the value for the total and bound susceptibilities. These quantities converge to a fixed value as the time progresses unlike for the sine-square pulse where it continues to decay. Similarly, the ionization probability $Q(t)$ attains a constant value due to the finite width of the pulse. Therefore, a linear extrapolation of the susceptibility versus the ionization probability is not feasible, see Fig. 4.12. The failure of the linear extrapolation is also supported by the data in Table 4.14 .

\section{Conclusions}

From this study, we have been able to draw three main conclusions. 
TABLE 4.14. Gaussian Pulse: Instantaneous $\chi$ versus $I\left(w / \mathrm{cm}^{2}\right)$

\begin{tabular}{ccccc}
\hline \hline$I\left(w / \mathrm{cm}^{2}\right)$ & $\chi\left(T_{\text {final }}\right)$ & $\chi$ Instant & Correlation & Standard Error \\
\hline $1 \cdot 10^{2}$ & 4.5885 & -92.4301 & -0.3722 & 80.6040 \\
$1 \cdot 10^{4}$ & 4.5885 & -9.6489 & -0.3678 & 81.9046 \\
$1 \cdot 10^{6}$ & 4.5885 & 6.1534 & 0.01872 & 28.4999 \\
$1 \cdot 10^{8}$ & 4.5885 & 4.7095 & 0.1618 & 0.3210 \\
$1 \cdot 10^{10}$ & 4.5886 & 4.5455 & 0.1635 & 0.03744 \\
$1 \cdot 10^{12}$ & 4.5917 & 4.5462 & 0.1630 & 0.03912 \\
$1 \cdot 10^{13}$ & 4.6210 & 4.5684 & 0.1580 & 0.04218 \\
$1.5 \cdot 10^{13}$ & 4.6381 & 4.5813 & 0.1558 & 0.04411 \\
$2 \cdot 10^{13}$ & 4.6562 & 4.5943 & 0.1569 & 0.04630 \\
$2.5 \cdot 10^{13}$ & 4.6758 & 4.6065 & 0.1671 & 0.04896 \\
$3 \cdot 10^{13}$ & 4.6974 & 4.6162 & 0.1949 & 0.05234 \\
$3.5 \cdot 10^{13}$ & 4.7218 & 4.6201 & 0.2508 & 0.05679 \\
$4 \cdot 10^{13}$ & 4.7515 & 4.6085 & 0.3582 & 0.06307 \\
$4.5 \cdot 10^{13}$ & 4.7873 & 4.5662 & 0.5286 & 0.06955 \\
$5 \cdot 10^{13}$ & 4.8251 & 4.5025 & 0.7011 & 0.06905 \\
$5.5 \cdot 10^{13}$ & 4.8692 & 4.4532 & 0.8093 & 0.06191 \\
$6 \cdot 10^{13}$ & 4.9331 & 4.4284 & 0.8492 & 0.05687 \\
$6.5 \cdot 10^{13}$ & 5.0098 & 4.4288 & 0.8131 & 0.06122 \\
$7 \cdot 10^{13}$ & 5.0709 & 4.4451 & 0.7018 & 0.07135 \\
$7.5 \cdot 10^{13}$ & 5.1259 & 4.4614 & 0.5277 & 0.08175 \\
$8 \cdot 10^{13}$ & 5.2224 & 4.4754 & 0.2722 & 0.09355 \\
$8.5 \cdot 10^{13}$ & 5.3478 & 4.4946 & -0.06714 & 0.1082 \\
$9 \cdot 10^{13}$ & 5.4107 & 4.5197 & -0.3836 & 0.1237 \\
$9.5 \cdot 10^{13}$ & 5.3959 & 4.5413 & -0.5858 & 0.1397 \\
$1 \cdot 10^{14}$ & 5.4364 & 4.5558 & -0.6954 & 0.1593 \\
$1.5 \cdot 10^{14}$ & 4.5238 & 4.8095 & -0.9347 & 0.4960 \\
$2 \cdot 10^{14}$ & -3.8445 & 5.2490 & -0.9585 & 1.0968 \\
$2.5 \cdot 10^{14}$ & -23.2516 & 5.8449 & -0.9681 & 1.8378 \\
$3 \cdot 10^{14}$ & -53.0440 & 6.5360 & -0.9742 & 2.5869 \\
\hline & & & &
\end{tabular}

First, with respect to the computation of the total and bound polarizations, we have found that the multidomain Legendre method with Crank-Nicolson time stepping is well suited. The method resolves the spatially oscillatory $R_{l}$ states and is sufficiently accurate to propagate on the time scale of the fast carrier wave. More importantly, the conservative nature of the algorithm minimizes amplitude errors which are not well tolerated by the calculation of the polarization.

Second, we have found that both the total and bound susceptibilities exhibit saturation for high intensity light. This proves therefore that it is not necessary to include bound-continuum transitions to observe this effect. Since the saturation occurs for both the Gaussian and sine-square pulses, it is also not particular to an electric field 
profile. The onset of the behavior however occurs for the total susceptibility at smaller intensities $I \sim 5 \cdot 10^{13}\left(\mathrm{w} / \mathrm{cm}^{2}\right)$ than for the bound susceptibility $I \sim 10^{14}\left(\mathrm{w} / \mathrm{cm}^{2}\right)$.

Regarding the determination of the instantaneous component of the susceptibility from a linear extrapolation of $\chi$ or $\chi_{b}$ versus the ionization probability $Q(t)$, we have determined that this procedure is quantitatively unreliable. Instead we have observed that this approach is highly sensitive to the shape of the electric field pulse and the magnitude of the ionization probability. For small values of $Q(t)\left(\sim 10^{-6}\right)$, it is not possible to distinguish simulation errors from the true values; while for large $Q(t)$, the extrapolation error is considerable. There is thus only a window of $Q$ values for which the extrapolation procedure is meaningful.

Considering future work, the application of a high order time stepping algorithm is an improvement which would greatly accelerate the computations [23]. Our simulations have revealed that the majority of the errors incurred are due to the second order time stepping. The evaluation of the commutator in the Strang splitting between the spatial derivative and the angular momentum coupling terms of the $R_{l}$ equations however presents a formidable challenge. Another application of the multidomain pseudospectral approach may be the simulation of multiple atoms. In this case, the savings in computer memory from the high order spacial discretization would be particularly advantageous. Finally, the next logical step in a further investigation is a comparison of the bound susceptibility computed from analytic means versus the numerically obtained curves. This may serve to establish to what extent the saturation is ionization induced or generated by high order nonlinear instantaneous susceptibility coefficients.

Acknowledgments. We wish to thank M. Nurhuda for candid and informative responses to our questions and M. Kolesik and W. Hoyer for useful discussions. This work was supported by the Air Force Office of Scientific Research under grant number F49620-02-1-0380.

\section{REFERENCES}

[1] E. Anderson, Z. Bai, C. Bischof, S. Blackford, J. Demmel, J. Du Croz, A. Greenbaum, S. Hammarling, D. A. McKenney and Sorensen, LAPACK Users' Guide, 3rd Ed., SIAM, Philadelphia, 1999.

[2] W. Becker, F. Grasbon, R. Kopold, D. B. Milosevic, G. G. Paulus and H. Walther, Abovethreshold ionization: from classical features to quantum effects, Adv. Atom. Mol. Opt. Phys., 48, 35-98, 2002.

[3] J. Boyd, Orthogonal rational functions on a semi-infinite interval, J. Comp. Phys., 70, 63-88, 1987.

[4] J. Boyd, Chebyshev and Fourier Spectral Methods, 2nd ed., Dover, New York, 2000.

[5] J. Boyd, C. Rangan and P. H. Bucksbaum, Pseudospectral methods on a semi-infinite interval with application to the hydrogen atom: a comparison of the mapped Fourier-sine method with Laguerre series and rational Chebyshev expansions, J. Comp. Phys., 188, 56-74, 2003.

[6] R. Boyd and Govind Agrawal, Contemporary Nonlinear Optics, Academic Press, San Diego, 1992.

[7] R. Boyd, Nonlinear Optics, Academic Press, San Diego, 1992.

[8] R. Boyd, Order-of-magnitude estimates of the nonlinear optical susceptibility, J. Mod. Opt., 46(3), 367-378, 1999.

[9] J. Brehm and W. Mullin, Introduction To The Structure Of Matter: A Course in Modern Physics, John Wiley, Sons, New York, 1989.

[10] K. Burnett, V. C. Reed and P. L. Knight, Atoms in ultra-intense laser fields, J. Phys. B.: At. Mol. Opt. Phys., 26, 561-598, 1993.

[11] C. Canuto, M. Y. Hussaini, A. Quarteroni and T. A. Tang, Spectral Methods in Fluid Dynamics, Section 2.3, Springer-Verlag, 1987. 
[12] R. V. Churchill, Fourier Series and Boundary Value Problems, McGraw-Hill Book Company, New York, 1941.

[13] P. Davis and P. Rabinowitz, Methods of Numerical Integration, Academic Press, New York, 1975.

[14] P. L. DeVries, Calculation of harmonic generation during the multiphoton ionization of the hydrogen atom, J. Opt. Soc. Am. B., 7(4), 517-520, 1990.

[15] P. L. DeVries, The time evolution of the hydrogen wave function in intense laser fields, Computer Physics Comm., 63, 95-99, 1991.

[16] J. H. Eberly, Q. Su and J. Javanainen, High-order harmonic production in multiphoton ionization, J. Opt. Soc. Am. B., 6(7), 1289-1298, 1989.

[17] B. Fornberg, Generation of finite difference formulas on arbitrarily space grids, Math. Comp., 51, 699-706, 1988.

[18] B. Fornberg, A Practical Guide To Pseudospectral Methods, Cambridge Univ. Press, New York, 1996.

[19] S. Geltman, Excitation and ionization of hydrogen atomics in ultra-intense fields, J. Phys. B: At. Mol. Opt. Phys, 27, 257-270, 1994.

[20] S. Gottlieb and C. Shu, Total variation diminishing Runge-Kutta schemes, Mathematics of Computation, 67(221), 73-85, 1998.

[21] J. S. Hesthaven, J. Rasmussen, L. Bergé and J. Wyller, Numerical studies of localized wavefields governed by the Raman-extended derivative nonlinear Schrödinger equation, J. Phys. A: Math. Gen., 30, 8207-8224, 1997.

[22] P. Kano, Development and analysis of high accuracy numerical methods for computational optics, Doctoral dissertation, U. of Arizona, 2005.

[23] A. Kassam and L. Trefethen, Fourth-order time-stepping for stiff PDEs, SIAM Journal on Scientific Computing, 26(4), 1214-1233, 2005.

[24] K. Kulander, Multiphoton ionization of hydrogen: a time-dependent theory, Phys. Rev. A, $35(1), 445-447,1987$.

[25] C. Mavriplis, Laguerre polynomials for infinite-domain spectral elements, J. Comp. Phys., 80, 480-488, 1989.

[26] P. Miloni and J. Eberly, Lasers, Wiley, New York, 1988.

[27] J. Moloney and A. Newell, Nonlinear Optics, Westview Press, Boulder, 2004.

[28] NIST, National Institute of Standards and Technology Reference on Constants, Units, and Uncertainty, http://physics.nist.gov/cuu/index.html, 2005.

[29] M. Nurhuda and F. H. Faisal, Numerical solution of the time-dependent Schrödinger equation for multiphoton processes: a matrix iterative method, Phys. Rev. A, 60, 4, 3125-3133, 1999.

[30] M. Nurhuda, A. Suda and K. Midorikawa, Saturation of dynamic nonlinear susceptibility of noble gas atom in intense laser field, RIKEN Review, 48, 40-43, 2002.

[31] M. Nurhuda, A. Suda and K. Midorikawa, Saturation of nonlinear susceptibility, J. Nonlin. Opt. Phys. \& Mat., 13(2), 301-313, 2004.

[32] H. X. Qiao, Y. C. Zou and Z. J. Zhang, High-order harmonic generation of hydrogen in intense laser field and strong magnetic field, Int. J. Mod. Phys. C, 15(4), 493-506, 2004.

[33] A. Ralston and P. Rabinowitz, A First Course in Numerical Analysis, 2nd Ed., Dover, Mineola, 2001.

[34] D. P. Shelton, Hyperpolarizability of the hydrogen atom, Phys. Rev. A., 36(7), 3032-3041, 1987.

[35] A. Taflov and S. Hagness, Computational Electrodynamics: The Finite Difference Timedomain Method, 2nd Ed., Artech House, Inc., Norwood, 2000.

[36] L. Trefethen, Finite Difference and Spectral Methods for Ordinary and Partial Differential Equations, unpublished text, http://web.comlab.ox.ac.uk/oucl/work/nick.trefethen/pdet ext.html, 1996.

[37] L. Trefetehn, Spectral Methods in MATLAB, SIAM, Philadelphia, 2000.

[38] G. von Winckel, The Mathworks File Exchange, http://www.mathworks.com/matlabcentral/ fileexchange/, 2004.

[39] J. A. C. Weideman and A. Cloot, Spectral methods and mappings for evolution equations on the infinite line, Comp. Meth. Appl. Mech. Engng., 80, 467-481, 1990.

[40] J. A. C. Weideman and S. C. Reddy, A MATLAB differentiation suite, ACM Transactions on Mathematical Software, 26(4), 465-519, 2000.

[41] Wolfram Research, Inc., Mathematica, Version 5.1, 2004.

[42] XMGRACE, Grace Development Team, http://plasma-gate.weizmann.ac.il/Grace/, 2004. 